\title{
Transient x-ray diffraction used to diagnose shock compressed Si crystals on the Nova laser
}

\author{
D. H. Kalantar, E. A. Chandler, J. D. Colvin, R. Lee, B. A. Remington, S. V. Weber, \\ and L. G. Wiley \\ Lawrence Livermore National Laboratory, Livermore, California 94550
}

A. Hauer

Los Alamos National Laboratory, Los Alamos, New Mexico 87545

J. S. Wark and A. Loveridge

Department of Physics, Clarendon Laboratory, University of Oxford, Oxford OX1 3PU, United Kingdom

B. H. Failor

Physics International, San Leandro, California 94577

M. A. Meyers

University of California at San Diego, La Jolla, California 92093

G. Ravichandran

California Institute of Technology, Pasadena, California 91125

(Presented on 9 June 1998)

Transient x-ray diffraction is used to record time-resolved information about the shock compression of materials. This technique has been applied on Nova shock experiments driven using a hohlraum x-ray drive. Data were recorded from the shock release at the free surface of a Si crystal, as well as from $\mathrm{Si}$ at an embedded ablator/Si interface. Modeling has been done to simulate the diffraction data incorporating the strained crystal rocking curves and Bragg diffraction efficiencies. Examples of the data and post-processed simulations are presented. () 1999 American Institute of Physics. [S0034-6748(99)62601-6]

\section{INTRODUCTION}

The response of a material to shock loading is typically described by a constitutive materials model such as the Steinberg-Guinan model. ${ }^{1}$ This is a semiempirical model that describes the material response using parameters that have been normalized against shock wave measurements of parameters such as the shear stress and yield stress. This model is an elastic-perfectly plastic model. When a stress is applied to a sample, it responds elastically up to the point where the stress exceeds the yield stress. As that point, it yields to plastic flow.

In fact, the material has a lattice structure not accounted for in such an empirical description. When the solid undergoes deformation at high pressure, stresses that occur at a lattice level result in the generation and subsequent propagation of dislocations. ${ }^{2,3}$ It is the rearrangement of the lattice structure by transport of these dislocations that is plastic material flow.

The number density of dislocations that are generated and the speed with which they propagate are dependent on the rate of the applied strain. The parameters defined in the constitutive materials model are determined from experiments such as those with strain rates on the order of

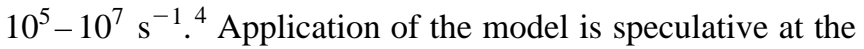
higher strain rates of $10^{7}-10^{9}$ that may be achieved with a laser driven shock experiment. As a result, it is important to characterize the shock response of materials at these high strain rates with a technique that probes the lattice structure itself. We use x-ray Bragg diffraction to achieve this.

In this article we describe the technique of dynamic diffraction and demonstrate its use for experiments done on the Nova laser ${ }^{5}$ in which single-crystal Si samples are shock compressed to pressures of up to 500 kbar using a hohlraum $\mathrm{x}$-ray drive. We describe the target geometry and two different shock experiments that we performed.

\section{DYNAMIC DIFFRACTION}

X-ray Bragg diffraction provides a measurement of the $2 d$ lattice spacing in a crystal structure. Here, $\mathrm{x}$ rays scat-

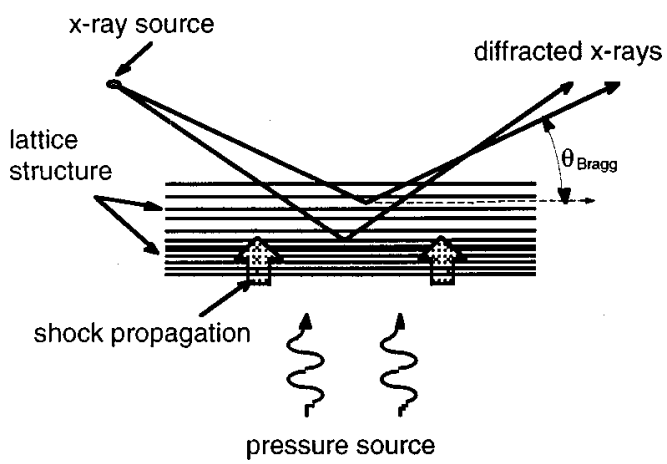

FIG. 1. Diagram showing the method of transient diffraction being used to probe a shock compressed lattice structure. 


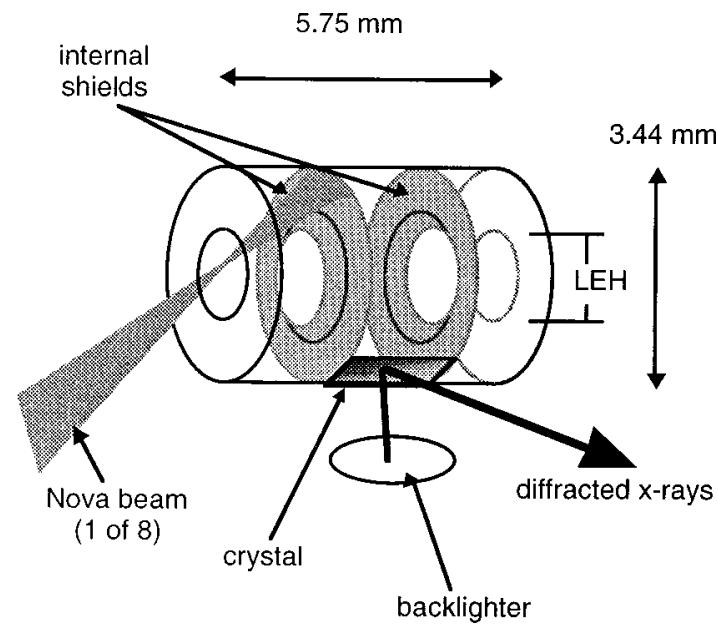

FIG. 2. Nova hohlraum target design used for transient x-ray diffraction experiments. The hohlraum has internal shields to prevent $\mathrm{x}$-ray preheat of the crystal sample. The crystal and backlighter foil are placed around the midplane of the target.

tered from the atoms in the lattice constructively interfere when the incident angle with respect to the lattice plane satisfies the Bragg condition:

$$
n \lambda=2 d \times \sin (\theta) .
$$

In this case, the $\mathrm{x}$ rays are diffracted at an angle $\theta$ equal to the incident angle (relative to the lattice planes) (Fig. 1).

When the lattice undergoes a deformation due to shock compression, the lattice spacing decreases. The Bragg diffraction condition is then met at a different angle determined by the new lattice spacing, provided the x-ray source is close enough to the sample that the crystal subtends a range of angles including the new Bragg angle. The shift in diffraction angle is recorded as a spatial shift in the diffraction signal.

Since the $\mathrm{x}$ rays that are typically used for Bragg diffraction are multi-keV, the depth in the crystal that can be probed may be tens of a $\mu \mathrm{m}$. If there is a range of densities and corresponding lattice spacings within this depth of the surface, we record a Bragg diffracted signal from the corresponding range of angles. In other words, we record a measure of the fraction of the material that is compressed at each density. By recording the signal with an x-ray streak camera, we obtain a time-resolved record of the compression in the crystal. For the case where there is a shock wave with a monotonic pressure profile (which results in a monotonic variation in material density for the case where there are no volumetric changes associated with a phase transition), the dependence of the diffraction signal on compression in principle can provide a measurement of the shock wave profile. ${ }^{6}$

\section{NOVA EXPERIMENT CONFIGURATION}

A cylindrical gold hohlraum target is used to generate a Planckian soft x-ray drive. The hohlraum target is shown in Fig. 2. It is $5.75 \mathrm{~mm}$ long and $3.44 \mathrm{~mm}$ in diameter with two internal shields. These shields divide the hohlraum into a laser heated cavity on each end, and an x-ray heated cavity in the center. Eight beams of the Nova laser are incident

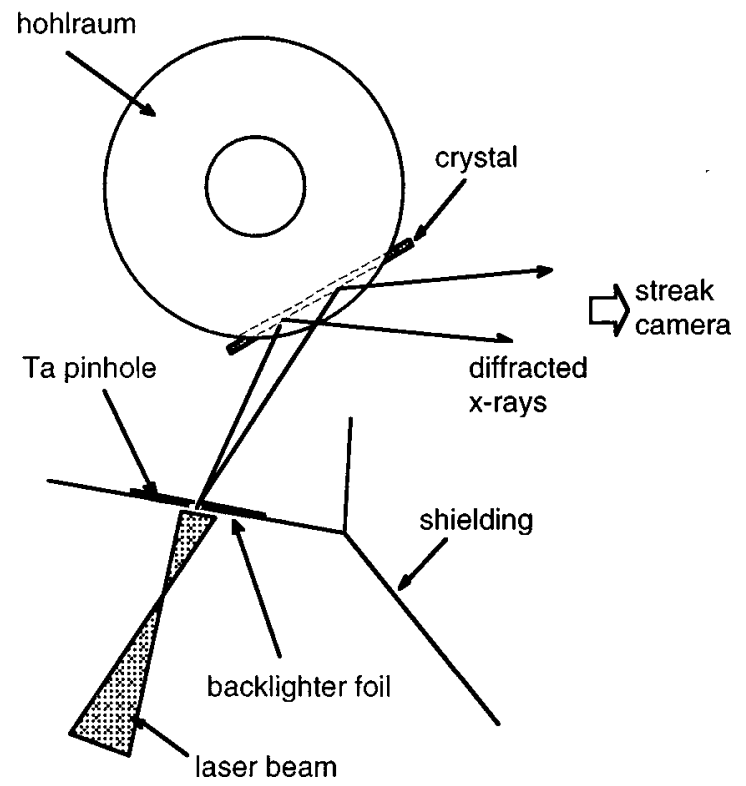

FIG. 3. Axial view of the target design used to probe the free surface of a shock compressed Si crystal.

through the two laser entrance holes at $50^{\circ}$ from the cylinder axis on the inner wall of the hohlraum. Gold $M$ band and thermal $x$ rays generated from the laser plasmas in the two laser heated cavities heat the central or x-ray heated cavity through the apertures in the internal shields, creating the Planckian x-ray drive.

The crystal sample is mounted over a hole in the side of the hohlraum at the midplane. The $\mathrm{x}$ rays in this cavity then ablatively drive a shock into the crystal, which we diagnose by x-ray diffraction. The internal shielding is designed so that a crystal sample placed over a hole in the hohlraum wall at the midplane has no direct line of sight to regions of the hohlraum that are directly illuminated with the high intensity laser. This prevents $2-4 \mathrm{keV} \mathrm{Au} M$-band emission from the laser heated spots from preheating the crystal prior to shock compression.

Two additional beams of Nova are used to generate the $\mathrm{x}$-ray source for Bragg diffraction. These beams are pointed onto a thin foil $(5-12 \mu \mathrm{m})$ such as vanadium. A Ta pinhole aperture is located behind the foil (Fig. 3), providing a small $(100 \mu \mathrm{m})$ source of $K$-shell line emission that is self filtered by the foil itself to provide a nearly monoenergetic backlighter spectrum. Note that by using a pinhole aperture to define the backlighter source size, we are able to generate a small backlighter source with a pulse length $>5 \mathrm{~ns}$. The expansion of the laser heated plasma creating the backlighter $\mathrm{x}$ rays is masked by the pinhole substrate.

The backlighter foil is located only about $2 \mathrm{~mm}$ from the crystal so that the crystal subtends an angle of up to about $25^{\circ}$ to the $\mathrm{x}$-ray source, allowing for a range of Bragg diffraction angles. The $\mathrm{x}$ rays diffracted from the crystal are recorded with an $\mathrm{x}$-ray streak camera. This camera is located with the photocathode $12 \mathrm{~cm}$ from the target, allowing us to collect nearly a $15^{\circ}$ range of diffraction angles from the target. A film holder is located in front of the streak camera to 


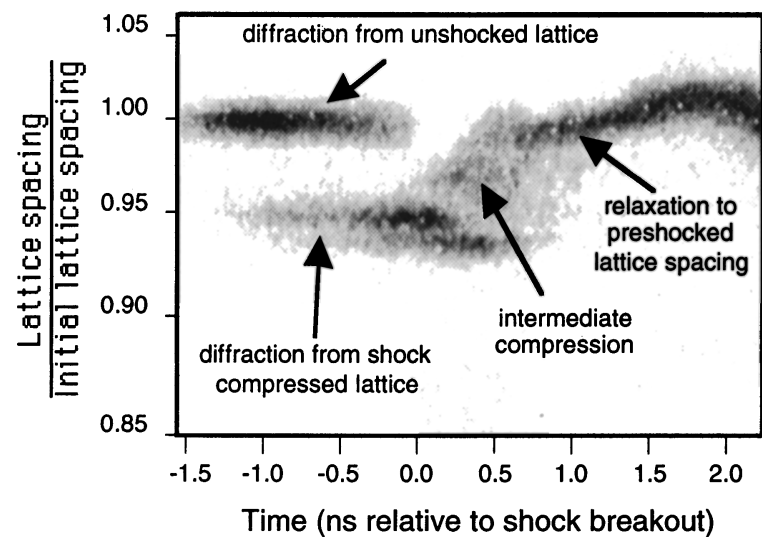

FIG. 4. Streak record of the x-ray diffraction from $\mathrm{Si}(111)$ shock compressed at $200 \mathrm{kbar}$.

record a time-integrated record of the Bragg diffracted $\mathrm{x}$ rays as well as the streak record.

A 4 ns square profile laser pulse delivers from 2.4 to 9.0 $\mathrm{kJ}$ of laser energy at $0.35 \mu \mathrm{m}$ into the hohlraum. An x-ray radiation field is created in the central region of the hohlraum, which ablatively drives a shock into the crystal. We probe the lattice structure by $\mathrm{x}$-ray diffraction as the shock propagates through the crystal. Since the backlighter, crystal, and streak camera are all positioned around the midplane of the hohlraum target, this technique is flexible in that it can be easily adapted for a variety of different crystals and lattice planes by matching the Bragg diffraction angle for the particular experiment.

We have recorded an x-ray diffraction signal prior to and during the shock breakout at the back (free) surface of a $\mathrm{Si}$ crystal. We have also recorded an x-ray diffraction signal from a Si crystal ablatively compressed with a low density $\mathrm{CH}(\mathrm{Br})$ ablator by probing through the ablator to the embedded $\mathrm{CH}(\mathrm{Br}) / \mathrm{Si}$ interface.

\section{BRAGG DIFFRACTION FROM Si(111)—FREE SURFACE}

Silicon at ambient pressure is a diamond cubic lattice. The $2 d$ spacing for the (111) lattice planes is $6.27 \AA$. A sample of Si $40 \mu \mathrm{m}$ thick oriented with the (111) lattice planes parallel to the surface was mounted over a hole on the midplane of the shielded hohlraum target. The hohlraum xray drive was used to shock compress the crystal. By using $3.2 \mathrm{~kJ}$ of laser energy in a $4 \mathrm{~ns}$ square pulse, a shock pressure of about $200 \mathrm{kbar}$ is generated in the $\mathrm{Si}$.

A V backlighter foil provided $K$-shell x rays of wavelength $2.38 \AA$, which Bragg diffract from the uncompressed (111) lattice planes at $22.3^{\circ}$. The backlighter was positioned so that the x-ray streak camera would collect the diffraction from the (111) lattice planes of solid density $\mathrm{Si}$ as well as from lattice planes compressed in one dimension by as much as about $6 \%$.

In Fig. 4(a) we show the streak record obtained from $\mathrm{Si}(111)$ using a $\mathrm{V}$ backlighter timed from 2.0 to 7.5 ns relative to the start of the hohlraum drive beams. This is plotted with axis scales to represent fractional 1D compression in

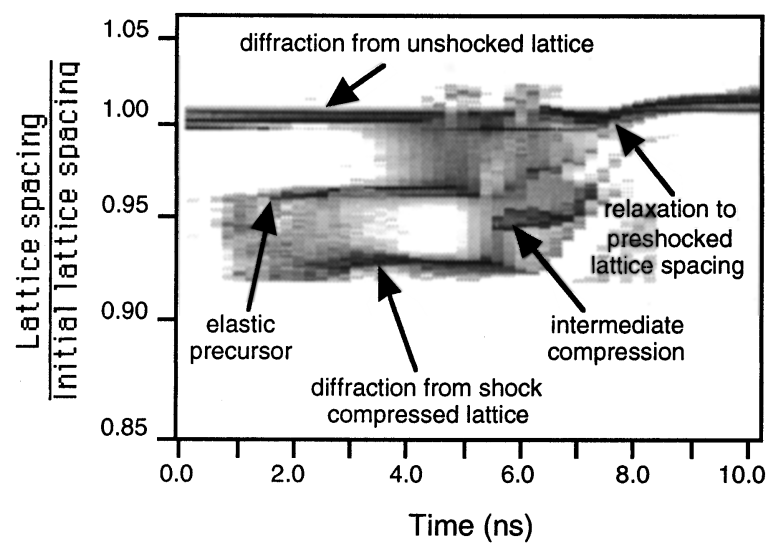

FIG. 5. Post-processed LASNEX simulation of the diffraction pattern from $\mathrm{Si}(111)$ shock compressed to $160 \mathrm{kbar}$.

lattice spacing normalized to the initial (111) lattice spacing as a function of time. Here, we observe diffraction from the uncompressed Si. We also observe diffraction from compressed $\mathrm{Si}$ as the shock propagates toward the back surface of the crystal. As the shock breaks out at the back surface, the signal from the unshocked material disappears since the full depth of the crystal is under compression. When the wave releases, the rarefaction returns the crystal to the initial density, characterized by the return of the diffraction signal corresponding to solid density Si.

This streak record potentially provides significant information about the shock propagation and lattice dynamics under compression at high strain rates. A vertical lineout through the data at a particular time provides a measurement of the distribution of lattice spacings within the probe depth, or a measurement of the shock front profile, convolved with the $\mathrm{x}$-ray absorption and Bragg diffraction efficiency.

Simulations were done using LASNEX $^{7}$ then post processed to generate simulated streaked $\mathrm{x}$-ray diffraction data. Strained crystal rocking curves were generated using a solution to the Takagi-Taupin equation, ${ }^{8-10}$ under a layer approximation, ${ }^{11}$ such that the strain is $1 \mathrm{D}$ and perpendicular to the diffracting planes. Then by stacking rocking curves calculated at different times, a simulated x-ray streak was generated. ${ }^{6}$ The resulting post-processed simulation is shown in Fig. 5 for the case where the $\mathrm{Si}$ is compressed at $160 \mathrm{kbar}$. Note that the simulation was done treating the $\mathrm{Si}$ as an isotropic fluid, which means that it does not include solid-solid phase transitions and associated volume changes that are observed under static compression at pressures $<200$ kbar. $^{12}$

\section{BRAGG DIFFRACTION FROM (111)-EMBEDDED INTERFACE}

We used the dynamic Bragg diffraction technique to measure shock timing and characterize the compression at the interface between a low density $\mathrm{CH}(\mathrm{Br})$ ablator and a $\mathrm{Si}$ crystal.

A $20 \mu \mathrm{m}$ thick layer of brominated polystyrene $[\mathrm{CH}(\mathrm{Br})]$ ablator was pressed in contact with a $40 \mu \mathrm{m}$ thick sample of (111) Si. Approximately $1000 \AA$ of $\mathrm{Al}$ was coated on the ablator, and this package was then mounted over a 


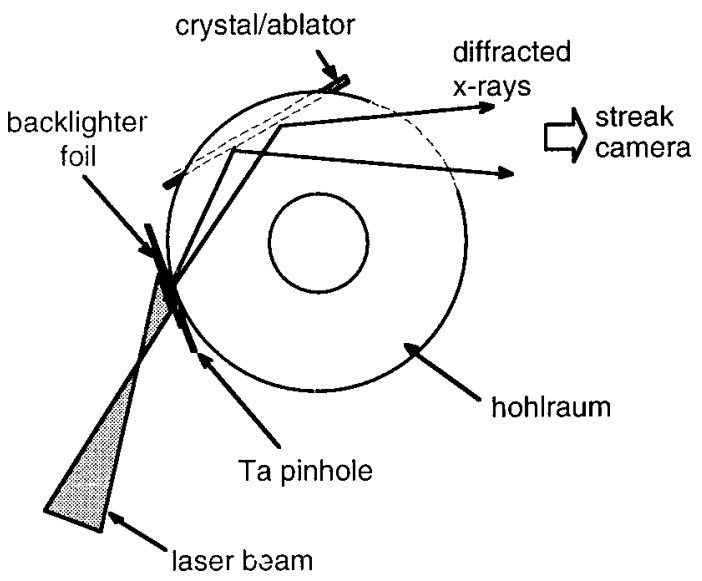

FIG. 6. Axial view of the target design used to characterize the shock propagation through a plastic ablator by $\mathrm{x}$-ray diffraction at the embedded plastic/Si interface.

hole on the midplane of the shielded hohlraum. A V backlighter foil was placed over a separate hole with a small $\mathrm{Ta}$ aperture $(100 \mu \mathrm{m})$ to provide a small x-ray backlighter source, as illustrated in Fig. 6. A separate slot was cut in the hohlraum to allow the diffracted $\mathrm{x}$ rays to be recorded with the x-ray streak camera.

The streaked diffraction data recorded from (111) $\mathrm{Si}$ driven with a low intensity square laser pulse is shown in Fig. 7. This pulse was about $1 / 2$ the laser power of the $4 \mathrm{~ns}$ pulse used for the free surface measurements, but the impedance mismatch at the $\mathrm{CH}(\mathrm{Br}) / \mathrm{Si}$ interface increases the pressure in the $\mathrm{Si}$. The shock pressure in the $\mathrm{Si}$ in this case is approximately $350 \mathrm{kbar}$. We observe the diffraction signal from the uncompressed $\mathrm{Si}$ starting when the backlighter source turns on at $t=3 \mathrm{~ns}$. After an additional $0.9 \mathrm{~ns}$, a shifted peak appears as the initial shock launched in the ablator arrives at the ablator/Si interface. There is an immediate compression by about $10 \%$ in the lattice spacing, followed by a second Bragg peak, which may indicate a solid-solid phase transition to a different lattice configuration. This is noted on the figure.

\section{SUMMARY}

We have applied the technique of transient x-ray diffraction to diagnose shock compression of materials on Nova using a hohlraum x-ray drive. This technique has been used to diagnose the shock compression of a Si crystal by recording the time-resolved Bragg diffraction pattern from the free surface of the crystal. We observed a shift in the diffraction

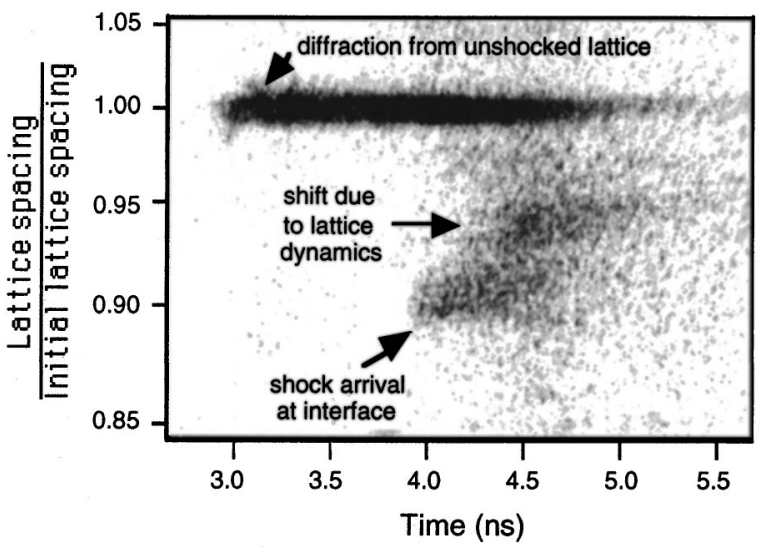

FIG. 7. Streak record of the x-ray diffraction from the ablator/Si(111) interface indicating shock arrival at the interface.

peak indicating a reduction in the lattice spacing of about $6 \%$ relative to the initial (111) Si lattice spacing, and we compare the time-resolved diffraction structure with postprocessed simulations. We have also used this technique to characterize the shock propagation time in a plastic ablator by recording the time the shock reaches an embedded ablator/crystal interface.

\section{ACKNOWLEDGMENTS}

The authors acknowledge the technical support of the Nova Operations and Target Fabrication groups. This work was performed under the auspices of the U.S. DOE by LLNL under Contract No. W-7405-ENG-48, and through the Materials Research Institute, LLNL, and the DOE Grants and University Use of Nova Programs.

${ }^{1}$ D. J. Steinberg, S. G. Cochran, and M. W. Guinan, J. Appl. Phys. 51, 1498 (1980).

${ }^{2}$ E. Zaretsky, J. Appl. Phys. 78, 1 (1995).

${ }^{3}$ L. C. Chhabildas and J. R. Asay, J. Appl. Phys. 50, 2749 (1979).

${ }^{4}$ J. W. Swegle and D. E. Grady, J. Appl. Phys. 58, 692 (1985).

${ }^{5}$ E. M. Campbell, J. T. Hunt, E. S. Bliss, D. R. Speck, and R. P. Drake, Rev. Sci. Instrum. 57, 2101 (1986).

${ }^{6}$ N. C. Woolsey and J. S. Wark, J. Appl. Phys. 81, 3023 (1997).

${ }^{7}$ G. B. Zimmerman and W. L. Kruer, Comments Plasma Phys. Control. Fusion 2, 51 (1975).

${ }^{8}$ S. Takagi, Acta Crystallogr. 15, 1311 (1962).

${ }^{9}$ S. Takagi, J. Phys. Soc. Jpn. 26, 1239 (1969).

${ }^{10}$ D. Taupin, Bull. Soc. Fr. Mineral. Cristallogr. 87, 469 (1964).

${ }^{11}$ C. R. Wie, T. A. Tombrello, and T. Vreeland, Jr., J. Appl. Phys. 59, 3743 (1985).

${ }^{12}$ M. Senoo, H. Mii, I. Fujishiro, and T. Fujikawa, Jpn. J. Appl. Phys. 15, 871 (1976); H. Olijnyk, S. K. Sikka, and W. B. Holzapfel, Phys. Lett. 103A, 137 (1984). 